\title{
Hemophilus Parainfluenzae Pneumonia With Huge IVC Thrombosis and Pulmonary Embolism in a Young Immunocompetent Male
}

\author{
Kuo-Hwa Chiang, ${ }^{\mathrm{a}, \mathrm{b}}$, , Nan-Chun $\mathrm{Wu}^{\mathrm{c}}$, Reng-Hong $\mathrm{Wu}^{\mathrm{d}}$
}

\begin{abstract}
Hemophilus parainfluenzae pneumonia is common in community acquired pneumonia, but Hemophilus parainfluenzae pneumonia with IVC thrombosis and pulmonary embolism is rare. The condition presents a significant diagnostic challenge. We report a 27year old immunocompetent male, who presented with recurrent pneumonia with Hemophilus parainfluenzae associated with deep vein thrombosis with huge inferior vena cava (IVC) thrombosis and pulmonary embolism. He was treated successfully with IVC filter placement and anticoagulant agents and the follow up angiography showed improvement of IVC thrombosis and pulmonary embolism.
\end{abstract}

Keywords: Hemophilus parainfluenzae pneumonia; IVC thrombosis; Deep vein thrombosis; Pulmonary embolism

Manuscript accepted for publication January 13, 2012

${ }^{a}$ Division of Chest Medicine, Department of Internal Medicine, Chi Mei Medical Center, Tainan, Taiwan (ROC)

${ }^{b}$ Department of Information Management, Chia Nan University of Pharmacy and Science, Tainan, Taiwan (ROC)

${ }^{\mathrm{c}}$ Department of Cardiovascular Surgery, Chi Mei Medical Center, Tainan, Taiwan (ROC)

${ }^{\mathrm{d}}$ Department of Radiology, Chi Mei Medical Center, Tainan, Taiwan (ROC)

${ }^{\mathrm{e}}$ Corresponding author: Kuo-Hwa Chiang, Division of Chest Medicine, Department of Internal Medicine, Chi Mei Medical Center, Tainan, Taiwan (ROC). Department of Information Management, Chia Nan University of Pharmacy and Science, No.901, Chun-Hua Rd. Yong Kang Dist, Tainan City 710, Taiwan (ROC).

Email: chiangkh2003@yahoo.com.tw

doi: $10.4021 / \mathrm{jmc} 511 \mathrm{w}$

\section{Introduction}

Respiratory tract infections remain a common cause of morbidity and mortality worldwide and pneumonia in particular is the leading cause of death due to infectious diseases in developed countries [1]. The microbiological aetiology of community acquired pneumonia (CAP) has changed over the last 20 - 30 years, and Hemophilus parainfluenzae has been implicated in pneumonia. Pillai et al. suggested that $H$ parainfluenzae can cause pneumonia with systemic features in the community, even in apparently immune competent individuals [1]. Pulmonary infection may increase the risk for venous thromboembolism (VTE). In previous literature Alikhan et al., suggested that acute respiratory infection may increase the risk of VTE [2]. Although the lifetime incidence of venous thrombosis is $0.1 \%$, it still remains a rare condition especially in patients below 30 years of age [3-5]. Deep vein thrombosis (DVT) accounts for approximately $66 \%$ of all VTE. Some DVT with pulmonary embolism (PE) may present with a similar clinical, radiological and laboratorial characteristics of CAP, represent a diagnostic challenge to the physician, especially in its difficulty in the differential diagnosis during treatment process [6]. Thrombosis of the inferior vena cava (IVC) with pulmonary embolism is a lifethreatening condition. It has similar aetiological factors to lower limb deep venous thrombosis (DVT). Hypercoagulability related to hematological or neoplastic abnormalities, venous stasis secondary to extraluminal pressure from tumours or inflammatory processes and vessel injury due to trauma have all been implicated as primary mechanisms in the pathophysiology of IVC thrombosis [7].

We present the case of atypical presentation of community acquired pneumonia associated with huge deep vein thrombosis with IVC thrombosis and pulmonary embolism and is, to the best of our knowledge, the first such case reported.

\section{Case Report}

A 27-year old male, non-smoker and with no known sys- 


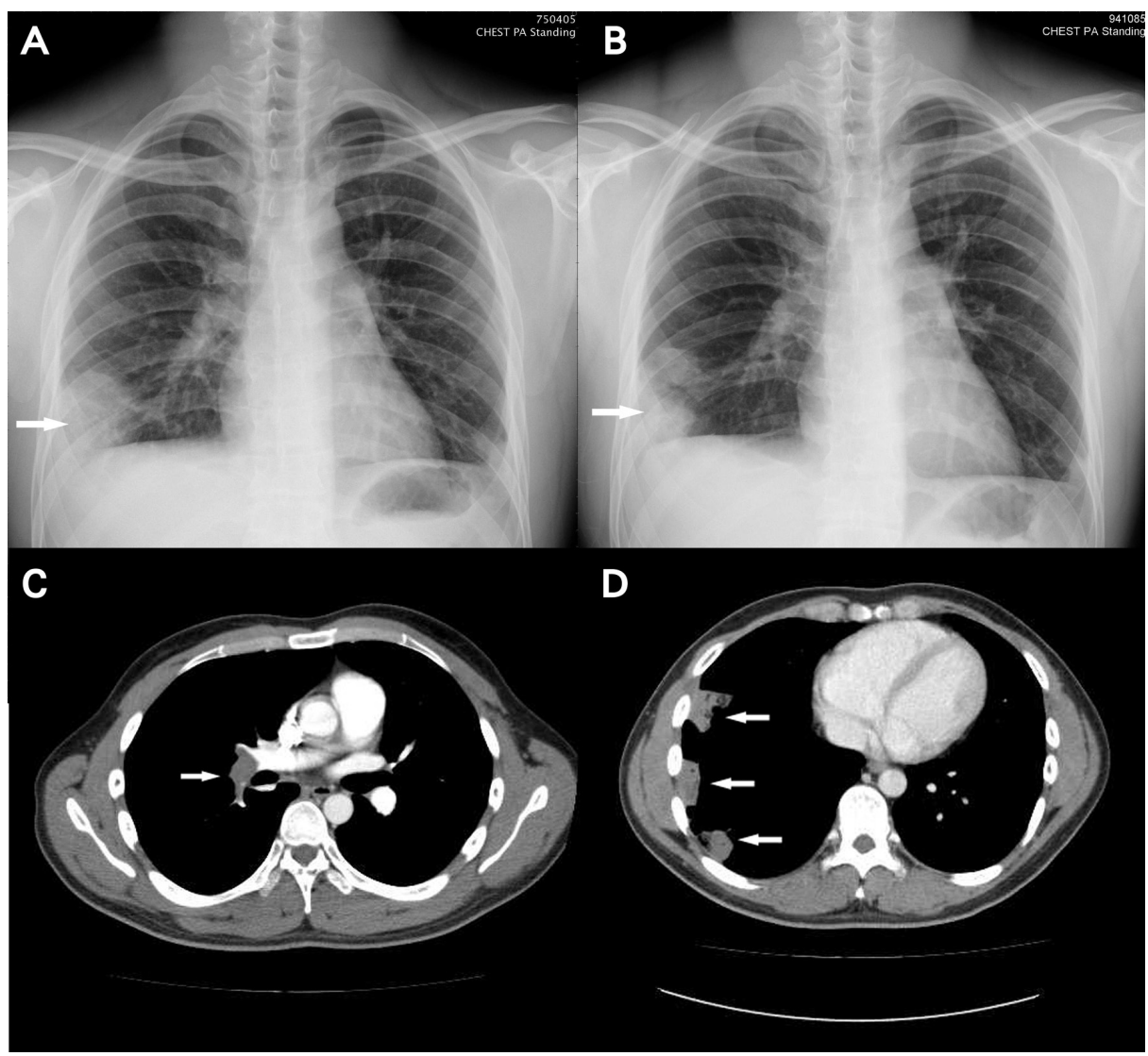

Figure 1. A: Chest radiograph on admission showing air space opacification over right lower lobe (arrow); B: Chest radiograph after empiric antibiotics treatment showing separated consolidative patch over right lower lobe (arrow) with slight resolution; C: CT angiogram showing pulmonary embolism of right main and descending arteries (arrow); $\mathrm{D}$ : chest CT demonstrating peripheral patches and consolidation in the right lower lung (arrow) corresponding to the consolidative patch seen on plain film.

temic disease, was admitted due to fever and right chest pain for 2 days, and had recent history of left lower lobe pneumonia discharged from other hospital one month prior to this admission. There was no thigh pain or lower limb swelling at initial presentation. On examination, he had a pyrexia of $38.5^{\circ} \mathrm{C}$ with a pulse rate of 96 beats/min and blood pressure of 126/74 mmHg. He had only slight chest pain during coughing otherwise no back pain no venous distension or pitting edema noted. The hematological analysis showed a platelet count of $274 \times 10^{3} / \mu 1$, white cell count of $16.8 \times 10^{3} /$ $\mu 1$, CRP: $74.5 \mathrm{mg} / \mathrm{L}$. The CXR revealed air space opacification over right lower lung (Fig.1A). Sputum culture revealed Hemophilus parainfluenzae, which was compatible with the pathogen isolate in other hospital. He was treated under the impression of pneumonia with suspicious parapneumonic effusion initially, adequate antibiotics treatment were used for severe sepsis and possible pathogen. His condition once improved and follow up CXR revealed separated consolidative patch over right lower lung filed with slight resolution (Fig. 1B). However left inguinal pain and left leg swelling developed several days after fever subsided. The sonogra- phy of the left lower extremities showed deep vein thrombosis of the left lower extremity. A cardiac echogram revealed moderate pulmonary hypertension with pulmonary arterial pressure of $55 \mathrm{mmHg}$. A contrast enhanced CT angiogram (Fig. 1C) scan from chest to pelvis and thigh revealed peripheral patches and consolidation in the right lower lung (Fig.1D) with abnormal filling defect over right main pulmonary artery and right descending pulmonary artery compatible with pulmonary embolism of right main and descending arteries(Fig. 2), complete thrombosis of left femoral to common iliac veins, and partial thrombosis of right common iliac vein to infrarenal IVC without other congenital IVC anomalies and no evidence of external compression of bilateral iliac veins(Fig. 3). Anti-cardiolipid antibody was $1.1 \mathrm{U} /$ $\mathrm{ml}$. RA factor $<10.6 \mathrm{IU} / \mathrm{ml}$, anti-nuclear antibody $40 \times(-)$ FA titer, p-ANCA (MPO) $<0.7 \mathrm{U} / \mathrm{ml}$, c-ANCA $($ PR3) $<0.6$ $\mathrm{U} / \mathrm{ml}$. The tumor markers were all negative as carcinoembryonic antigen (CEA): $1.7 \mathrm{ng} / \mathrm{ml}$, CA 19-9:21.0 U/ml, alphafetoprotein (AFP): $1.1 \mathrm{ng} / \mathrm{ml}$.

An extensive discussion regarding the risk and benefits of treatment planning was performed. The patient was ini- 


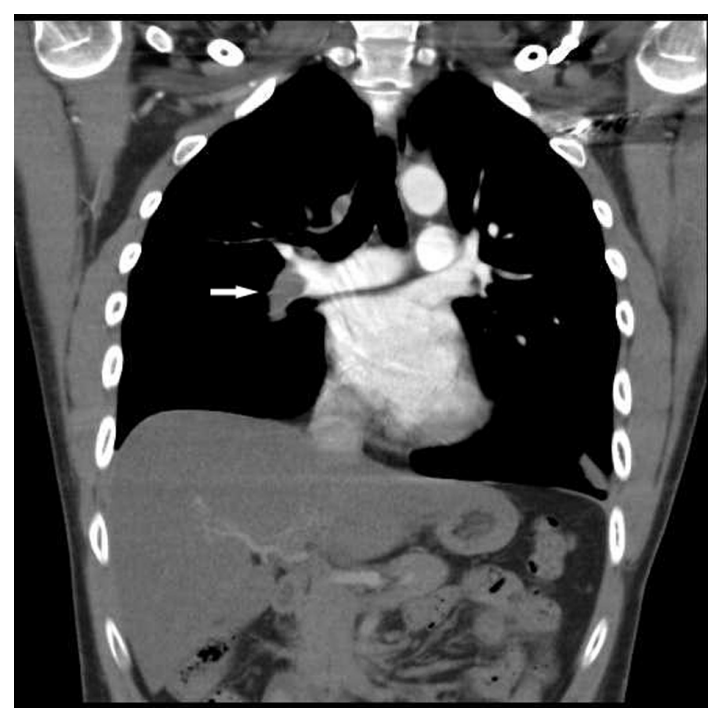

Figure 2. CT angiogram showing abnormal filling defect over right main pulmonary artery and right descending pulmonary artery (arrow) compatible with pulmonary embolism of right main and descending pulmonary arteries.

tially placed on a heparin drip and intentional radiology was consulted. A venocavogram was performed(Fig. 4A) and he was found to have a filling defect in IVC and right common iliac vein, and non-opacification of left iliac vein. A COOK Celect IVC filter was deployed at infrarenal IVC without significant tilting (Fig. 4B). The patient tolerated the procedure well, and anticoagulants agent with heparin, and urokinase were given. His condition improved after 7th day in ICU, and the follow up angiography revealed improvement of

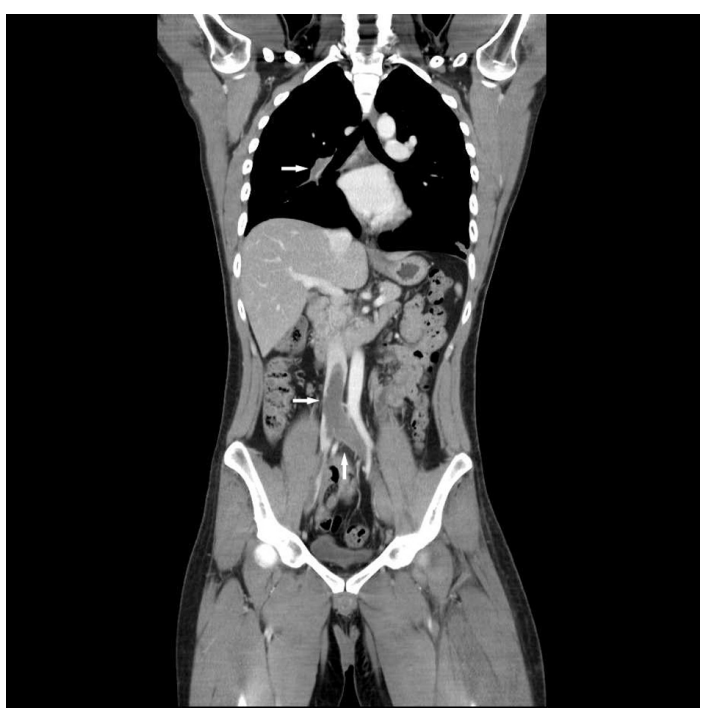

Figure 3. CT angiogram showing complete thrombosis of left femoral to common iliac veins, and partial thrombosis of right common iliac vein to infrarenal IVC (lower arrow).

IVC thrombosis and pulmonary embolism.

\section{Discussion}

We present this unusual case of recurrent pneumonia with rapidly progressing IVC thrombosis and pulmonary embolism in an immune competent young male. It demonstrates that unusual infectious pattern or noninfectious diseases

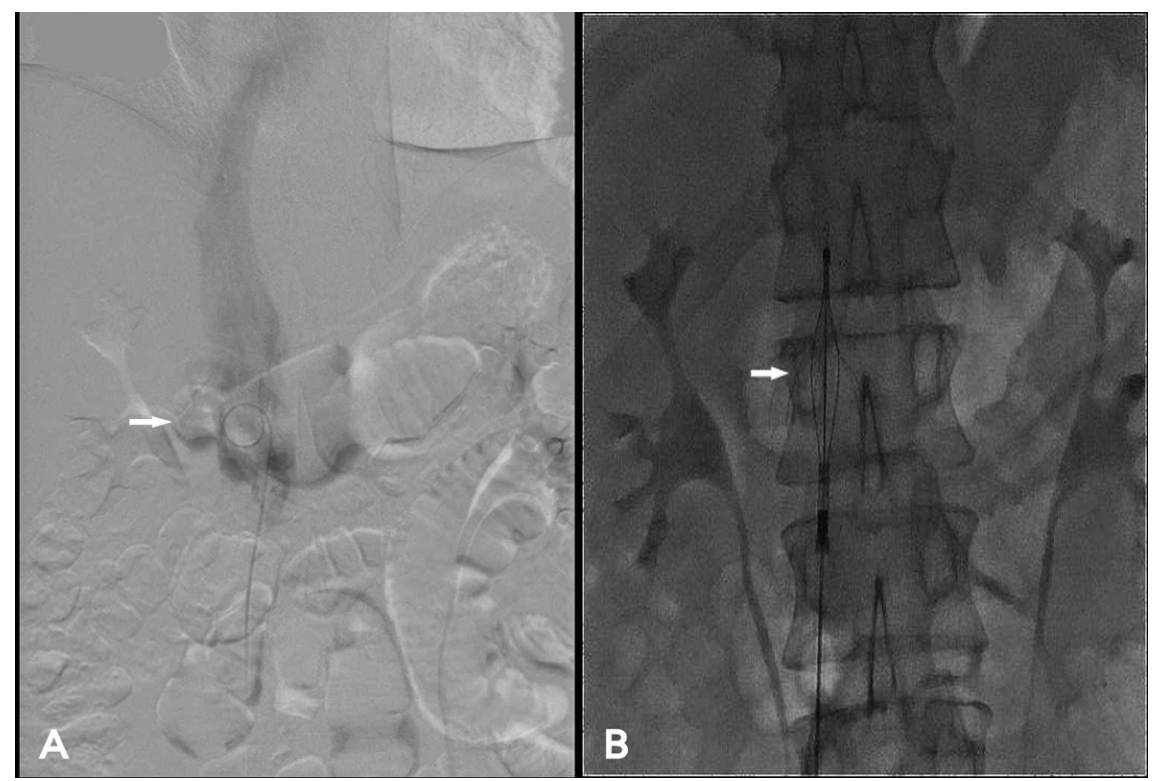

Figure 4. A: Venocavogram showing a filling defect in IVC (arrow) and right common iliac vein, and nonopacification of left iliac vein; B: A COOK Celect IVC filter deployed at infrarenal IVC (arrow). 
should always be suspected and included in the differential diagnosis of patients with presumptive diagnosis of pneumonia but who present with treatment failure or disease progression, especially young patients or those without comorbidity $[5,6]$. The first step toward those diagnostics is to constantly be aware of their possible presence.

Pulmonary infection may increase the risk for venous thromboembolism (VTE). In previous literature Alikhan et al., reported that acute respiratory infection was an independent risk factors in the generation of venous thrombosis [2]. H parainfluenzae pneumonia associated with DVT and IVC thrombosis complicated with pulmonary embolism is extremely rare. To the best of our knowledge, this is the first case ever reported.

Pulmonary embolism is an insidious disease with protean manifestation requiring high index of suspicion especially in young patients [5]. PE can present with cough, fever, Creactive protein elevation and pulmonary opacity, so that bacterial pneumonia could represent a confounding diagnosis $[5,6]$. In such condition the diagnosis of PE is difficult to obtain during initial stage of community acquired pneumonia before empiric antibiotics treatment. The classical presentation of IVC thrombus varies according to the level of the thrombosis with up to $50 \%$ of patients presenting with bilateral lower extremity swelling and dilatation of superficial abdominal vessels. However, some asymptomatic subjects might have had subclinical thrombosis that no one was able to diagnose before some complicated situation [7-9].

In our case, there is no leg pain, no swelling during initial presentation. The coagulation screen showed negative for anti-phospholipid antibody, RA factor, and anti nuclear antibody. It ruled out the possibility of certain acquired deficient. Additionally, the serological study also showed negative for $\mathrm{p}$ - ANCA and c-ANCA, which further ruled out the possibility of antineutrophil cytoplasm antibody (ANCA)associated systemic vasculitis such as Wegener's Granulomatosis. It is possible that acute pulmonary infection predisposed the thrombotic event.

Neoplasms have a strong relationship with IVC thrombosis because of hypercoagulability. Thrombosis of the inferior vena cava from extraluminal obstruction has been described in a variety of neoplasms and in inflammatory conditions such as pancreatitis, pelvic inflammatory disease, puerperal sepsis, and hepatic abscesses [7]. Reports of IVC thrombosis secondary to compression by abdominal aortic aneurysms, retroperitoneal fibrosis, and retroperitoneal hematomas also exist [7]. Following ultrasonic identification of an ileo-femoral thrombosis especially when bilateral, CT or MRI imaging, are required to delineate IVC anatomy and ascertain proximal extent of the thrombus [8]. Chest CT scans should extend up to the renal veins as this will identify common IVC anomalies [10]. Concurrent occlusion of the common iliac(s) as well as the IVC, with distal extension of thrombus leads to clinical symptomatology in patients with both normal and abnormal IVC anatomy [11]. We therefore performed CT scan from the thoracic inlet down to pelvis and popliteal area. However the chest and pelvis CT of this patient has ruled out the above possibility. Following exclusion of May Thurner syndrome and other pathologies, it is possible that in this patient the thrombus was a primary event associated with pneumonia. The diagnosis of this patient was therefore of $H$ parainfluenzae pneumonia associated with DVT and IVC thrombosis complicated with pulmonary embolism. Awareness of hemophilus parainfluenzae active role in thrombosis is an important point for suspect embolism and to avoid pitfalls in none resolving or relapsing pneumonia. From our case, acute Hemophilus parainfulenza infection can be the cause or precipitating factor of thromboembolic events in immunocompetent patients. For this reason we suggest considering $\mathrm{PE}$ as a differential diagnosis in patients presenting with atypical presentation of community acquired pneumonia especially in those patients with risk factors.

In conclusion, an unusual presentation of pneumonia in young patient merit special attention, and a high index of suspicion is warranted for early detection of IVC thrombus with pulmonary embolism in the young patient with lower back and limb pain, swelling of the lower limbs, with a concurrent rise in inflammatory markers and pyrexia.

\section{Grant of Support}

None.

\section{Conflict of Interests}

We declare that the content has not been previously published and has been submitted solely to Journal of Medical Cases. All authors have participated sufficiently in the intellectual content and the analysis of data. Each author has reviewed the final version of the manuscript and approved it for publication. We declare no conflict of interest.

\section{References}

1. Pillai A, Mitchell JL, Hill SL, Stockley RA. A case of Haemophilus parainfluenzae pneumonia. Thorax. 2000;55(7):623-624.

2. Alikhan R, Cohen AT, Combe S, Samama MM, Desjardins L, Eldor A, Janbon C, et al. Risk factors for venous thromboembolism in hospitalized patients with acute medical illness: analysis of the MEDENOX Study. Arch Intern Med. 2004;164(9):963-968.

3. Anderson FA, Jr., Wheeler HB, Goldberg RJ, Hosmer DW, Patwardhan NA, Jovanovic B, Forcier A, et al. A population-based perspective of the hospital incidence 
and case-fatality rates of deep vein thrombosis and pulmonary embolism. The Worcester DVT Study. Arch Intern Med. 1991;151(5):933-938.

4. Nordstrom M, Lindblad B, Bergqvist D, Kjellstrom T. A prospective study of the incidence of deep-vein thrombosis within a defined urban population. J Intern Med. 1992;232(2):155-160.

5. Rosendaal FR. Thrombosis in the young: epidemiology and risk factors. A focus on venous thrombosis. Thromb Haemost. 1997;78(1):1-6.

6. Alves dos Santos JW, Torres A, Michel GT, de Figueiredo CW, Mileto JN, Foletto VG, Jr., de Nobrega Cavalcanti MA. Non-infectious and unusual infectious mimics of community-acquired pneumonia. Respir Med. 2004;98(6):488-494.

7. Giordano P, Weber K, Davis M, Carter E. Acute throm- bosis of the inferior vena cava. Am J Emerg Med. 2006;24(5):640-642.

8. McAree BJ, O’Donnell ME, Boyd C, Spence RA, Lee B, Soong CV. Inferior vena cava thrombosis in young adults--a review of two cases. Ulster Med J. 2009;78(2):129-133.

9. Chee YL, Culligan DJ, Watson HG. Inferior vena cava malformation as a risk factor for deep venous thrombosis in the young. Br J Haematol. 2001;114(4):878-880.

10. Tsuji Y, Inoue T, Murakami H, Hino Y, Matsuda H, Okita Y. Deep vein thrombosis caused by congenial interruption of the inferior vena cava--a case report. Angiology. 2001;52(10):721-725.

11. Raju S, Hollis K, Neglen P. Obstructive lesions of the inferior vena cava: clinical features and endovenous treatment. J Vasc Surg. 2006;44(4):820-827. 